\title{
Publisher Correction to: A Microscopic Model for a One Parameter Class of Fractional Laplacians with Dirichlet Boundary Conditions
}

C. Bernardin, P. Gonçalves \& B. Jiménez-Oviedo

\author{
Correction to: Arch. Rational Mech. Anal. \\ https://doi.org/10.1007/s00205-020-01549-9
}

Due to typesetting mistakes, in equations 2.1 and 2.6 the 1 was incorrect.

On page 17, the second equation of Theorem 3.2 was incorrect. The original article has been corrected.

Publisher's Note Springer Nature remains neutral with regard to jurisdictional claims in published maps and institutional affiliations. 


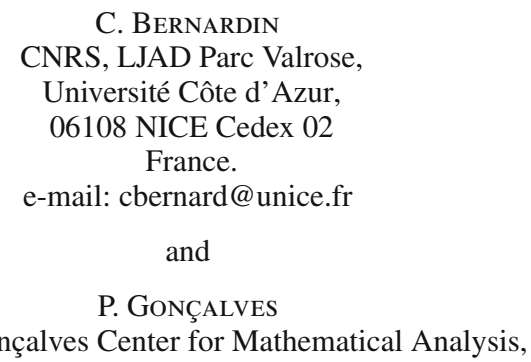

Patrícia Gonçalves Center for Mathematical Analysis, Geometry and Dynamical Systems, Instituto Superior Técnico, Universidade de Lisboa, Av. Rovisco Pais, 1049-001 Lisbon

Portugal.

e-mail: pgoncalves@tecnico.ulisboa.pt

and

UMS 839 (CNRS/UPMC),

Institut Henri Poincaré,

11 Rue Pierre et Marie Curie,

75231 Paris Cedex 05

France.

and

B. JIMÉNEZ-OVIEDO

Escuela de Matemática, Facultad de Ciencias Exactas y Naturales, Universidad Nacional de Costa Rica,

Heredia

Costa Rica.

e-mail: byron.jimenez.oviedo@una.cr

Published online October 8, 2020

(C) Springer-Verlag GmbH Germany, part of Springer Nature (2020) 